\title{
Détermination des efforts exercés par le vent sur un arbre
}

Alain FAURE \& Jacques PELLET

I.N.R.A., Station de Science du Sol, Centre de recherches d'Avignon, F 84140 Montfavet

RÉSUMÉ

La poussée du vent dans la superstructure d'un arbre entraîne un fléchissement du tronc dans le sens du vent et un allongement de ces fibres extérieures situées au vent.

Des essais in situ et des simulations de l'action du vent par une traction horizontale ont été effectués.

La mesure, par jauges extensométriques, de cet allongement permet théoriquement d'apprécier non seulement le point d'application résultant de la poussée, mais également le module d'élasticité de l'arbre. Ces essais se sont heurtés à des difficultés d'isolation thermique des capteurs, toutefois les résultats obtenus ouvrent des perspectives intéressantes.

La mesure de la flèche d'un arbre à une hauteur donnée a permis de montrer que le point d'ancrage résultant de l'arbre se situe à une profondeur qu'il a été possible d'apprécier avec une bonne précision.

Une fois connus les éléments de réduction des efforts exercés sur un arbre par le vent (résultante, point d'application de cette résultante et point d'ancrage), il est maintenant opportun de comprendre la façon dont ces efforts sont repris par le système racinaire. C'est la seule condition pour arriver à déterminer la résistance des arbres à l'arrachement sous l'action du vent.

Mots clés additionnels : Module d'élasticité, ancrage, console, jauge extensométrique, flèche.

\section{Determination of the effects of wind strength on a tree.}

The effect of wind strength on the superstructure of a tree is to create a bending moment on the trunk and an elongation of the external windward fibres.

Wind action simulations have been made by horizontal traction on the tree. Measurement of elongation with strain gauges theoretically gave the position of the strength barycentre and the Young's modulus of the tree. Measurements of bending of the trunk at a given height gave the depth of the equivalent anchorage center. Having analyzed these different elements reducing stress under wind pressure, it is necessary to understand how these stresses are taken up by the root system. This is an essential condition for determining the resistance of the tree to uprooting by the wind.

Additional key words : Young's modulus, anchorage, bracket, strain gauge, bending.

\section{LISTE DES SYMBOLES}

(m) Distance au point d'ancrage équivalent du point d'application de F.

b (m) Profondeur du point d'ancrage équivalent.

$\varepsilon \quad(/)$ Déformation relative d'un élément de matériau.

E (Pa) Module d'élasticité linéaire du matériau.

F (N) Composante horizontale de l'effort exercé sur un arbre.

H (m) Hauteur par rapport au sol du point d'application de l'effort.

$\mathrm{H}_{1}$ (m) Hauteur par rapport au sol du point de mesures.

I $\left(\mathrm{m}^{4}\right)$ Moment d'inertie de la section droite du tronc, considéré comme cylindre à base circulaire.
R (m) Rayon moyen de la section droite du tronc d'un arbre.

$\mathrm{R}_{\mathrm{c}} \quad$ (m) Rayon de courbure de la déformée de la ligne moyenne du tronc.

y (m) Flèche du tronc à la hauteur $\mathrm{H}_{1}$.

\section{INTRODUCTION}

La compréhension des causes de l'arrachage accidentel des arbres par le vent implique nécessairement l'étude de l'ancrage de l'arbre. Cet ancrage contribue, d'une part, à l'équilibre statique de la partie aérienne et, d'autre part, à la résistance au basculement lorsque l'arbre est soumis à l'action du vent. Parmi le nombre de questions qui se 
présentent et auxquelles il conviendrait de répondre, se trouve le mystère de la répartition spatiale de cet ancrage dans le sol, soute autre condition de nutrition ou de résistance du sol restant inchangée. En effet, en ne considérant que le comportement mécanique des acteurs en présence - vent, arbre, racines, sol - on est tenté de penser que l'enracinement optimal, c'est-à-dire celui qui donne une résistance maximale, n'a pas la même répartition spatiale selon que le vent a une direction dominante ou qu'il n'en a pas. GRACE (1977) cite HenwOOd (1973), SENN (1923) et NAVEZ (1930) qui ont observé l'existence d'un contrefort généré par le tronc du côté du vent dominant.

Les réponses à ce genre de questions, et il y en a bien d'autres, impliquent que soient déterminées les caractéristiques des efforts et des moments que le vent exerce sur l'obstacle, ce qui fait prendre en compte également des critères aérodynamiques. Il est clair, en effet, que la connaissance des efforts appliqués à la partie aérienne de l'arbre est indispensable car ce sont cux qui, en définitive, peuvent être la cause des désordres. Toutefois, il convient de connaître également la façon dont ces efforts sont transmis aux racines par le tronc. Alors seulement les processus d'interaction des racines et du sol sauront donner la réponse concernant l'équilibre de l'ensemble, phénomène dont ils détiennent la clef.

Des mesures in situ sont présentées et discutées ici. Il a été possible de dégager un certain nombre de démarches possibles. Chacune d'elles sera présentée en montrant les difficultés rencontrées et les succès que l'on peut espérer en obtenir.

\section{DESCRIPTION DES EFFORTS EXERCÉS PAR LE VENT SUR UN ARBRE}

Sur une section horizontale à la base du tronc, l'ensemble des efforts agissant sur la partie aérienne se réduit à 2 forces et 2 moments :

- les efforts verticaux dus au poids de la structure aérienne et des surcharges saisonnières (feuilles, neige) ont pour résultante un effort normal à la section considérée.

- les efforts horizontaux dont l'intensité est variable et qui sont exercés par le vent lui-même. Il est théoriquement possible de déterminer à chaque instant la force équivalente $F$ ainsi que la hauteur de son point d'application. Cette force engendre un effort tranchant dans la section considérée.

- le moment fléchissant créé par cette force $F$, auquel il convient d'ajouter le moment dû au poids de l'arbre lorsque celui-ci est incliné.

- un moment de torsion engendré par une dissymétrie éventuelle, soit de la structure de l'arbre, soit de l'action du vent sur cette siructure par rapport au plan vertical contenant le tronc et parallèle à la direction instantanée du vent. En première approximation, ce moment de torsion sera considéré comme négligeable bien qu'existant dans la plupart des cas.

Les 2 grandeurs les plus intéressantes apparaissent donc être le moment fléchissant et l'effort tranchant. FRASER \& GARDINER (1967) et SOMERVILLE (1979) se sont surtout intéressés au moment fléchissant lors de leurs essais, en exerçant une force horizontale au centre de gravité de l'arbre. Par ailleurs, l'utilisation de la formule classique de l'aérodynamique donnant la force $F$ exercée par un vent de vitesse d'écoulement $V$ n'en définit pas le point d'application.
Enfin, aucune de ces démarches n'évoque la possibilité de déterminer la position, soit du centre instantané de rotation, soit du point d'encastrement de l'arbre. L'étude du comportement d'un arbre sous l'action d'une force horizontalc apporte des éléments de réponses concernant non seulcment la détermination des propriétés élastiques du tronc, mais également les conditions d'ancrage dans le sol.

\section{A. Fléchissement d'un arbre sous l'action d'une force horizontale}

\section{Principe}

Puisque le poids de l'arbre n'intervient pratiquement pas lorsque le fléchissement est de faible valeur, l'étude envisagée est assimilable à celle d'une console non pesantc, soumise en son extrémité libre à un effort $\mathrm{F}$ perpendiculaire à sa ligne moyenne. Parmi les inconnues à déterminer figure la position du point d'encastrement.

L'idée de base de ces essais est d'utiliser les propriétés ćlastiques de l'arbre lui-même de 2 façons complémentaires. D'une part, par mesures de la flèche du tronc, d'autre part, par mesures des déformations de la fibre extérieure du tronc, lors de la flexion qu'il subit. Il est ainsi possible de déterminer les caractéristiques élastiques du bois, la hauteur $\mathrm{H}$ au-dessus du sol du point d'application de la résultante et enfin la profondeur du point d'ancrage équivalent, c'est-à-dire le point d'encastrement. On supposera négligeables les efforts mis en jeu par les racines et le sol audessus du point d'ancrage.

\section{a) La flèche du tronc}

Au point $\mathrm{A}$ d'abscisse $\mathrm{x}$, la flèche $\mathrm{y}$ (notations de la fig. 1) d'une console de moment d'inertie I constant, de module d'élasticité $E$ et soumise à une force $F$ est donnée par la relation théorique de l'élasticité linéaire :

$$
y=\frac{F}{6 E I}\left[2 a^{3}-3(a-x) a^{2}+(a-x)^{3}\right] .
$$

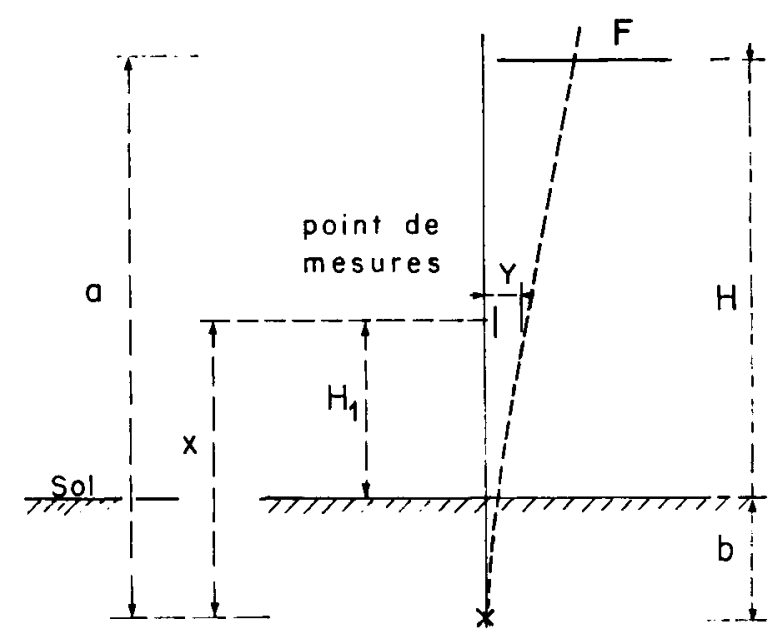

Figure 1

Notations utilisées.

Conventional notations.

Deux méthodes différentes (cf. 2) ont été utilisćes pour déterminer expérimentalement les valeurs de la flèche d'un arbre pour des efforts horizontaux donnés. 


\section{b) La déformation de la fibre supérieure}

La déformation $\varepsilon$ au point $A$ de la fibre supérieure de la console est donnée par la relation théorique de l'ćlasticité linéaire :

$$
\varepsilon=\frac{\mathrm{R}}{\mathrm{R}_{\mathrm{c}}}
$$

équation dans laquelle $\mathrm{R}$ est le rayon de la section droite de la console au point $A$ et $R_{c}$ le rayon de courburc de la déformée de la ligne moyenne en ce point dont l'expression en fonction des dérivées de y est :

$$
\frac{1}{\mathrm{R}_{\mathrm{c}}}=\frac{\mathrm{y}^{\prime \prime}}{\left(1+\mathrm{y}^{\prime 2}\right)^{3 / 2}} .
$$

D'après l'équation (1), il vient :

$$
y^{\prime}=\frac{F}{2 E I}(2 a-x) x \quad \text { et } \quad y^{\prime \prime}=\frac{F}{E I}(a-x) .
$$

Or, le module d'élasticité du bois a pour ordre de grandeur $10 \mathrm{GPa}$ et les arbres étudiés ont un rayon $\mathrm{R}$ de l'ordre de $0,1 \mathrm{~m}$, ainsi le produit $\mathrm{E} I$ est de l'ordre de $8.10^{5} \mathrm{~N} . \mathrm{m}^{2}$. Dans la gamme des efforts $\mathrm{F}$ mis en jeu lors de nos essais, soit $3 \mathrm{kN}$ au maximum, la valeur maximale de $y^{\prime}$ est environ de $2.10^{-3}$. Nous pouvons donc considérer que la quantité $y^{\prime 2}$ est négligeable devant 1 et que l'équation (3) devient :

$$
\frac{1}{R_{c}}=y^{\prime \prime} .
$$

Ainsi l'équation (2) pcut s'écrire :

$$
\varepsilon=\frac{R F}{E I}(a-x)=\frac{R F}{E I}\left(H-H_{1}\right) .
$$

L'utilisation simultanée de 2 capteurs de déformations à 2 emplacements différents sur le tronc donne, pour une même valeur de $F, 2$ équations où les 2 inconnues sont $F$ et $\mathrm{H}$, intensité de la force du vent et hauteur du point d'application de cette force.

\section{Matériels et méthodes}

\section{a) Mesure de la flèche}

\section{- Méthode de la cornière}

Une cornière métallique, rigide, de quelques $\mathrm{cm}$ de longueur est fixée sur le tronc à la hauteur $\mathrm{H}_{1}$ au-dessus du sol et soit $\mathrm{x}=\mathrm{b}+\mathrm{H}_{1}$ sa distance à l'cncastrement. Cette cornière est fixéc de telle sorte qu'une de ses faces soit perpendiculaire et l'autre parallèle à la direction de la force. Lors de la simulation de l'action du vent, les déplacements $d_{1}$ et $d_{2}$ de ces 2 faces selon leur normale (fig. 2) donnent la flèche y du tronc à la hauteur $\mathrm{H}_{1}$ avec

$$
y=\left(d_{1}^{2}+d_{2}^{2}\right)^{1 / 2} .
$$

\section{- Méthode du chariot}

La flèche de l'arbre en un point $\mathrm{A}$, situé à une hautcur $\mathrm{H}_{1}$ choisie, est appréciée par la mesure du déplacement horizontal d'un chariot roulant avec des frottements négligeables sur un bâti placé dans l'axe de l'action de la force (fig. 3). Le chariot est relié au point A par une tige

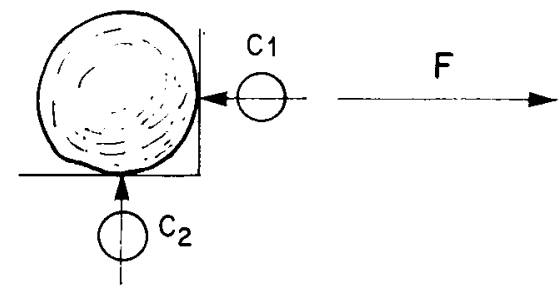

Figure 2

Méthode de la cornière: place des comparateurs par rapport à la direction du vent.

Angle bar method: location of comparators with regard to wind direction.

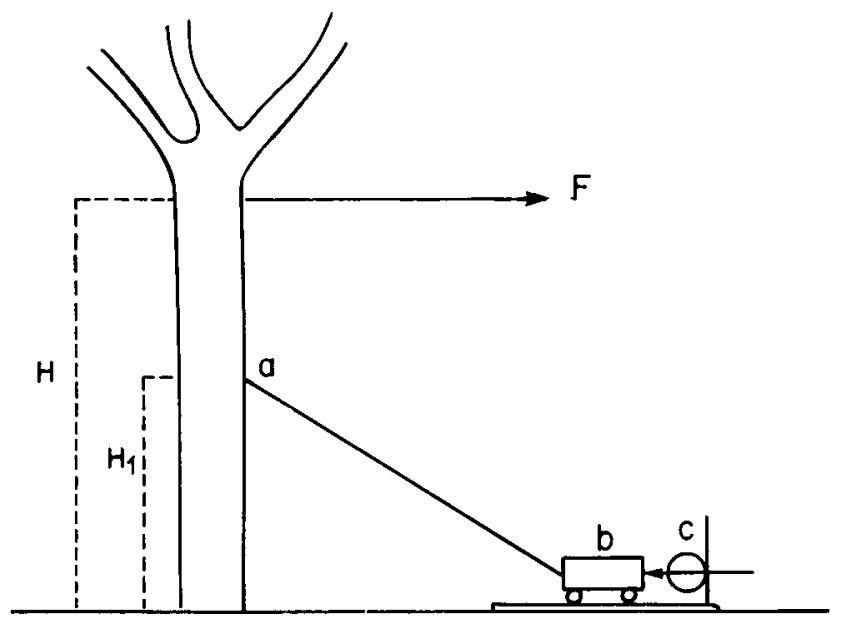

Figure 3

Méthode du chariot: dispositif

$a$ : charnière $b$ : chariot $c$ : comparateur.

Waggon method: apparatus

$a$ : hinge $b$ : waggon $c$ : comparator.

métallique rigide s'articulant en $\mathrm{A}$ au moyen d'un gond à axe horizontal. La valeur de la flèche est obtenue par lecture directe sur comparateur du déplacement du chariot.

\section{b) Mesure de la déformation. Méthode du capteur}

Le collage d'une jauge d'extensométrie sur une partic lissée et nettoyée du tronc lui-même présente l'avantage d'une lecture directe de la déformation de la fibre sur laquelle elle est collée. Toutefois, cette méthode présente de nombreux inconvénients de mise en ouvre et une grande consommation de jauges. Aussi a-t-il été adopté le principc de l'utilisation de capteurs dont la maniabilité est plus grande.

Les capteurs utilisés (fig. 4) sont basés sur le même principe que ceux conçus par FERRAND (1982). Un corps rigide $\mathrm{A}$ est muni à une de ses extrémités d'une lamelle souple, servant de palpeur, sur laquelle est collée une jauge d'extensométrie. L'autre extrémité du corps rigide est muni de 2 supports de fixation. Lors de l'utilisation du capteur, les 2 supports et la pointe du palpeur sont légèrement enfoncés dans le tronc de telle sorte que le segment (ab) soit parallèle aux génératrices du tronc. Les variations de longueur du segment $(a b)$ de la fibre du tronc mettent en flexion la lamelle du palpeur. Le capteur n'est donc opérationnel que lorsque, par étalonnage préalable, la relation entre la réponse de la jauge et la déformation de la 


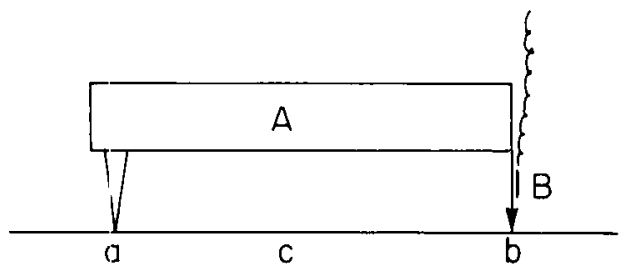

Figure 4

Type de capteur

$A$ : corps rigide $B$ : palpeur $C$ : support.

Extensometric gauge

$A$ : rigid body $B$ : gauge $C$ : tree.

fibre a pu être établie. Ce type de capteur présente toutcfois l'inconvénient de perdre une puissance de 10 dans la précision de la réponse, par rapport à la mesure directe de la déformation.

\section{Résultats numériques}

\section{a) Méthode de la cornière}

Deux essais ont été effectués sur un érable de diamètre moyen $\varnothing=15,4.10^{-2} \mathrm{~m}$ à 2 hauteurs de mesure. La hauteur $H$ est égale à $2 \mathrm{~m}$ dans les 2 cas. Nous retrouvons expérimentalement ce que la théorie de l'élasticité linéaire laissait espérer: il y a proportionnalité (fig. 5) entre les déplacements, et donc la flèche, et l'intensité de la force

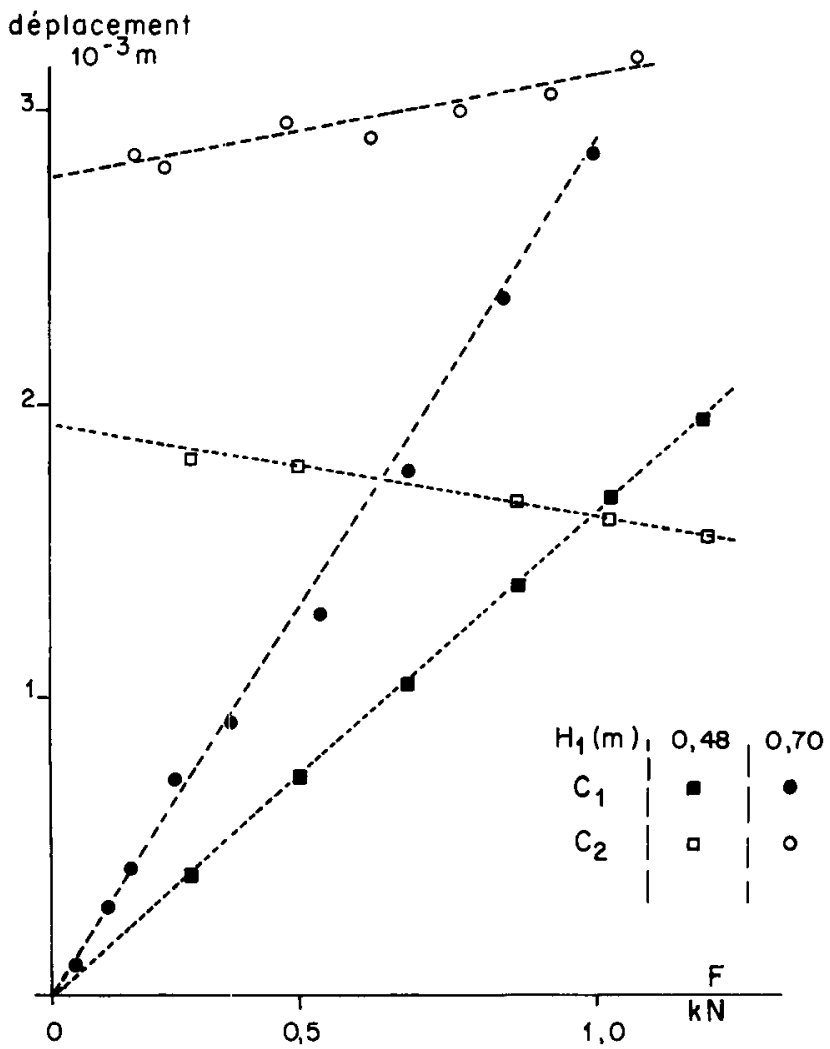

Figure 5

Méthode de la cornière: fléchissement d'un arbre en fonction de l'effort horizontal.

$C_{1}:$ comparateur sous le vent.

$\mathrm{C}_{2}$ : comparateur perpendiculaire au vent.

Angle bar method: tree bending versus horizontal stress.

$C_{1}:$ comparator in line with wind.

$C_{2}$ : comparator perpendicular to the wind. appliquée. Ainsi, les hypothèses initiales sur le caractère parfait de l'élasticité de l'arbre sont compatibles avec l'expérimentation. Les résultats sont résumés dans le tableau 1 :

\section{TABLEAU 1}

Méthode de la comière: résultats expérimentaux sur érable. Angle bar method: experimental data from maple.

\begin{tabular}{cccc}
\hline \hline Essai & $\begin{array}{c}\mathrm{H}_{1} \\
(\mathrm{~m})\end{array}$ & $\begin{array}{c}\mathrm{a}-\mathrm{x} \\
(\mathrm{m})\end{array}$ & $\mathrm{y}_{\text {cxp }} \times 10^{6} \times \mathrm{F}^{-1}$ \\
\hline 1 & 0,70 & 1,30 & 3,08 \\
2 & 0,48 & 1,52 & 1,81 \\
\hline
\end{tabular}

L'utilisation des valcurs expérimentales de la flèche y et des quantités $\mathrm{a}-\mathrm{x}$ dans la relation (1) donne 2 équations à 2 inconnues a et $\mathrm{E}$, dont la résolution conduit aux valeurs numériques suivantes:

$$
\begin{array}{ll}
\mathrm{a}=2,20 \mathrm{~m} & \begin{array}{l}
\text { soit une profondeur du point d'ancrage } \\
\text { équivalent de } 0,20 \mathrm{~m}
\end{array} \\
\mathrm{E}=9,0 \mathrm{GPa} &
\end{array}
$$

Ces résultats seront analysés dans le paragraphe 4 .

\section{b) Méthode du chariot}

Deux séries d'essais ont été effectuées pour 2 valeurs différentes de la hauteur $\mathrm{H}_{1}$. Dans chaque série, l'intensité de la force, appliquée à l'aide d'une corde reliće à un dynamomètre et un palan, a été moduléc. Trois essais ont été effectués dans chaque série, en faisant varier la hauteur $\mathrm{H}$ par rapport au sol du point d'application de la force. Les résultats (fig. 6) obtenus sur un platane de $14.10^{-2} \mathrm{~m}$ de diamètre montrent clairement la linéarité de la flèche en fonction de l'effort appliqué.

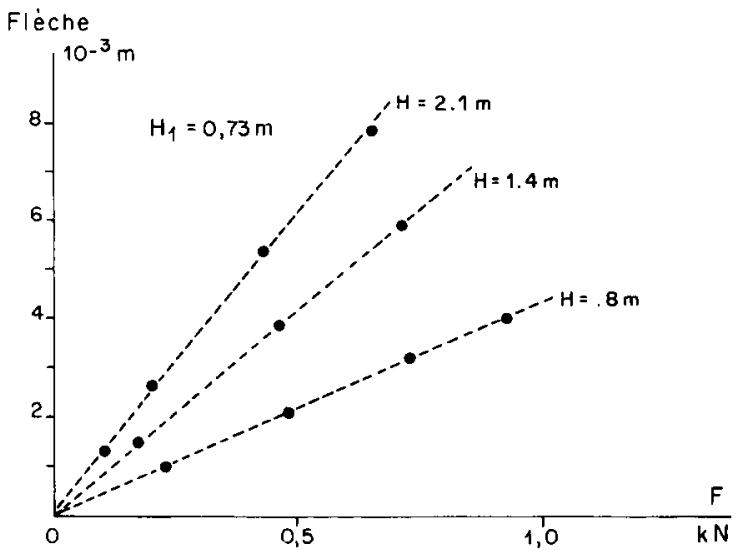

Figure 6

Méthode du chariot: fléchissement d'un arbre en fonction de l'effort horizontal.

Waggon method: tree bending versus horizontal force.

Les résultats sont rassemblés dans le tableau 2.

Du fait que, pour chaque essai, la hauteur $\mathrm{H}$ du point d'application de la force a été modifiée, il convient de 
TABLEAU 2

Méthode du chariot: résultats expérimentaux sur platane.

Waggon method: experimental data from plane tree.

\begin{tabular}{cccc}
\hline \hline $\begin{array}{c}\mathrm{N}^{\circ} \\
\text { essai }\end{array}$ & $\begin{array}{c}\mathrm{H}_{1} \\
(\mathrm{~m})\end{array}$ & $\begin{array}{c}\mathrm{H} \\
(\mathrm{m})\end{array}$ & $y_{\exp } \times 10^{6} \times \mathrm{F}^{-1}$ \\
\hline 1 & 0,73 & 0,80 & 4,4 \\
2 & 0,73 & 1,40 & 8,3 \\
3 & 0,73 & 2,10 & 12,2 \\
4 & 0,145 & 0,60 & 0,6 \\
5 & 0,145 & 1,30 & 1,0 \\
6 & 0,145 & 2,00 & 1,5 \\
\hline
\end{tabular}

remplacer $\mathrm{a}$ par $\mathrm{b}+\mathrm{H}$ dans l'ćquation (1) où $\mathrm{b}$ devient l'inconnue. Il vient :

$$
\begin{aligned}
y=\frac{F}{6 E I} & {\left[2 b^{3}+3\left(H+H_{1}\right) b^{2}+\right.} \\
& \left.+6 H_{1} b+H^{2}\left(3 H_{1}-H\right)+\left(H-H_{1}\right)^{3}\right] .
\end{aligned}
$$

Cette équation, appliquée aux 6 essais, donne 6 relations numériques aux inconnues $\mathrm{b}$ et $\mathrm{E}$.

Il vient : $\mathrm{E}=3,4 \mathrm{GPa}$

$$
\mathrm{b}=0,16 \mathrm{~m} \text {. }
$$

\section{c) Méthode du capteur extensométrique}

Les essais ont été conduits sur l'érable utilisé au $\S$ a, en conservant $\mathrm{H}=2 \mathrm{~m}$ comme hauteur du point d'application de la force horizontale. Le capteur a été placé à la hauteur $\mathrm{H}_{1}=1 \mathrm{~m}$ au-dessus du sol, le rayon moyen du tronc de l'érable est $7,7 \cdot 10^{-2} \mathrm{~m}$.

Les résultats (fig. 7) donnent la relation expérimentale suivante :

$$
\varepsilon=0,43 \cdot 10^{-6} \mathrm{~F} \text {. }
$$

A l'aide de l'ensemble de ces valeurs numériques, l'équation théorique (4) donne :

$$
\mathrm{E}=6,5 \mathrm{GPa} \text {. }
$$

\section{Discussion et conclusion}

Les résultats obtenus par ces diverses méthodes apparaissent cohérents en ce sens que les valeurs du module d'élasticité obtenues sont dans la gamme de ce qui est

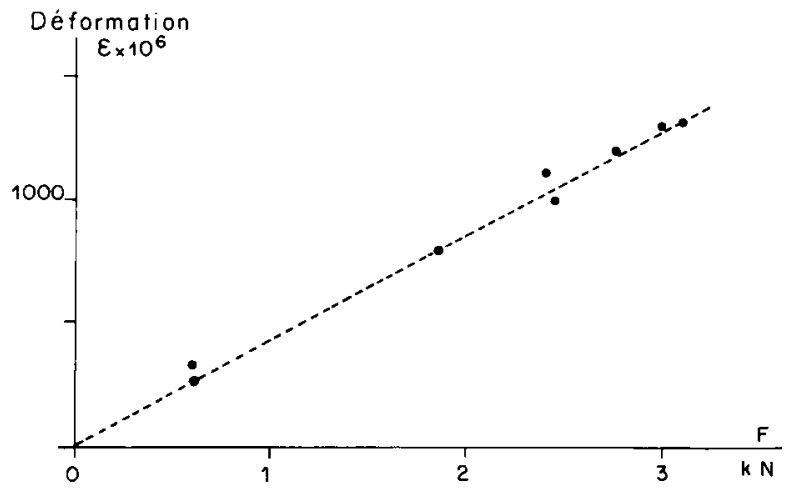

Figure 7

Déformation de la fibre du tronc à la hauteur $H_{1}=1 \mathrm{~m}$ sous l'action d'un effort horizontal.

Windward elongation of trunk fibre at $H_{1}=1 \mathrm{~m}$ under horizontal force. habituellement trouvé. BUCUR (1981), par exemple, a obtenu sur des éprouvettes de hêtres des modules d'élasticité de l'ordre de $11 \mathrm{GPa}$, valeurs légèrement plus élevées dont une raison pourrait être un état de siccité plus élevé du matériau utilisé lors des essais, hormis le fait que les essences sont différentes.

La précision des résultats obtenus, particulièrement avec la méthode dite du chariot, permet d'affirmer qu'unc sollicitation horizontale de faible intensité s'exerçant sur un arbre donne à celui-ci un comportement comparable à celui d'une console dont l'encastrement est situé en profondeur. Dans les cas présentés, cette profondeur est de $0,16 \mathrm{~m}$ pour un jeune platane et de $0,20 \mathrm{~m}$ pour un jeune érable, avec des modules d'élasticité respectifs de 3,5 et $7 \mathrm{GPa}$ environ.

Il apparaît également que la profondeur de l'encastrement pourrait être indépendante de la hauteur du point d'application de la force, tout au moins dans la gamme des hauteurs et des efforts choisie. Cette dernière conclusion ne s'imposait pas intuitivement avant la réalisation des essais.

\section{B. Résultante F de l'action du vent sur un arbre}

La force d'entraînement $F$, ou traînée, qui s'exerce sur un arbre est celle qui s'exerce sur un corps plongé dans un fluide s'écoulant à la vitesse $\mathrm{V}$ et a pour expression :

$$
\mathrm{F}=\frac{1}{2} \mathrm{C}_{\mathrm{d}} \mathrm{V}^{2} \mathrm{~S} \rho
$$

dans laquelle

$\mathrm{C}_{\mathrm{d}} \quad$ est le coefficient de traînée, fonction de la forme du corps (sans dimension)

$\rho \quad$ est la masse volumique du fluide, exprimée en $\mathrm{kg} \cdot \mathrm{m}^{-3}$

$\mathrm{S}$ est l'aire de la section du corps, orthogonale à l'écoulement.

GUYOT (1978) propose une détermination des efforts exercés par le vent sur un brise-vent. Cette détermination, à la suite des travaux de Plate (1971), Hagen \& Skidmore (1971a), BEAN et al. (1975) notamment, prend en compte la porosité du brise-vent dont l'influence sur le coefficient de traînée ne peut être établie que de façon empirique. Il est évident que les calculs proposés, concernant des surfaces poreuses relativement rigides, ne sont pas directement applicables au cas des arbres dont la mobilité du feuillage rend difficile la détermination de la porosité de l'écran.

D'autre part, la prise en compte de la distribution expérimentale ou théorique de la vitesse horizontale du vent en fonction de l'altitude en présence d'un obstacle n'est pas l'objet de cet article contrairement aux excellentes études effectuées par HAGEN \& SKIDMORE (1971b) et par SEGUIN \& GIGNOUX (1974).

Le coefficient de traînée d'un arbre a été déterminć expérimentalement par mesure directe et simultanée de la vitesse du vent et de la flèche de l'arbre en un point donné. Cette méthode, utilisée sur le platane déjà étudié, c'est-àdire dont le point d'encastrement est connu, permet de définir les éléments de réduction principaux des efforts exercés par le vent sur une section horizontale à la base du tronc et d'apprécier les caractéristiques de ces efforts au point considéré comme le point d'encastrement.

\section{Matériel et méthode}

Les conditions des essais in situ ont rendu inadaptée l'utilisation du capteur extensométrique par temps agité du fait que la jauge qu'il porte a une grande sensibilité aux 


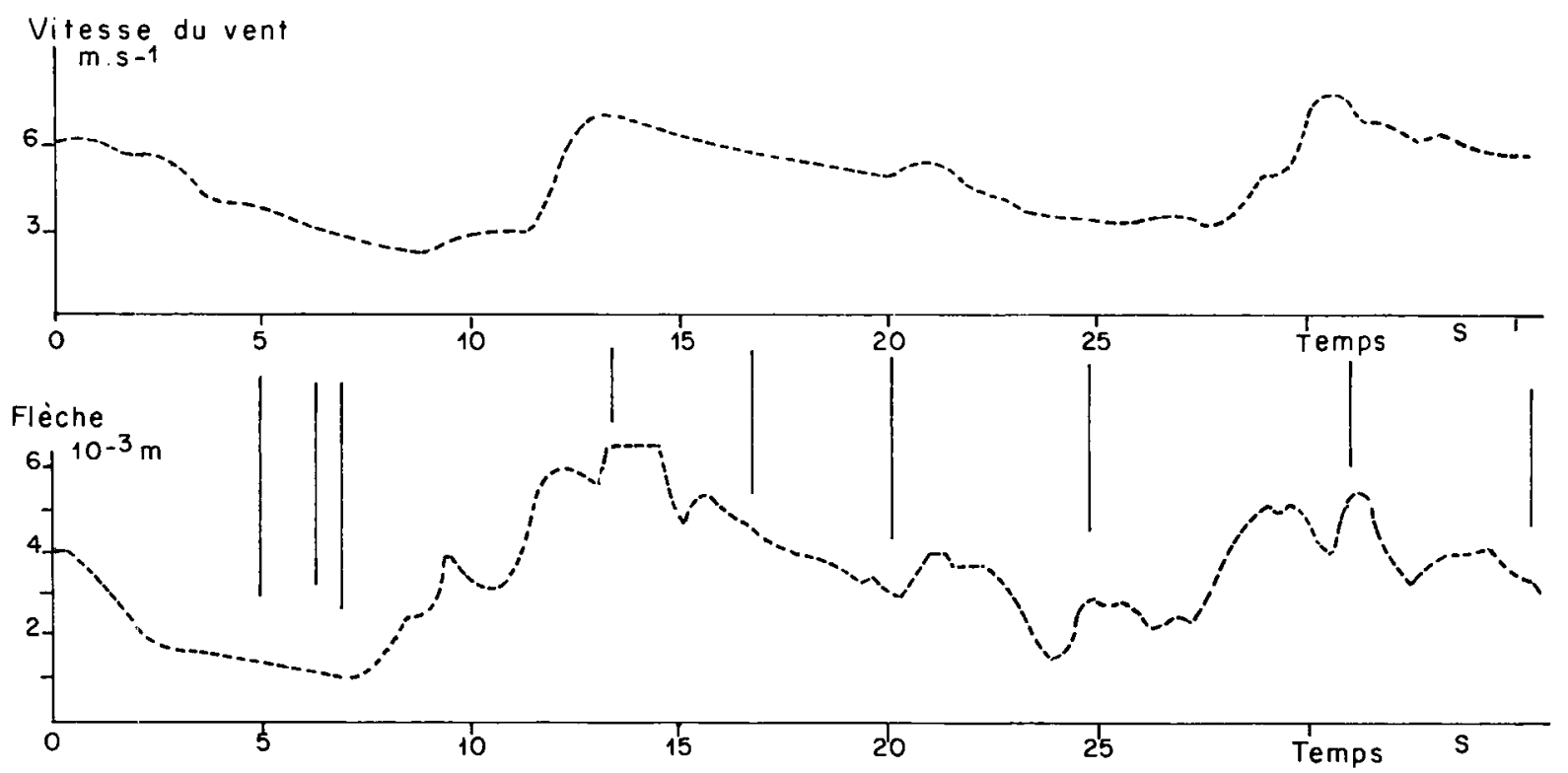

Figure 8

Enregistrements simultanés de la vitesse du vent et de la flèche d'un arbre à la hauteur $H_{t}=0,73 \mathrm{~m}$.

variations thermiques. Aussi, seules les mesures de la flèche ont été effectuées. Nous avons retenu la méthode dite du chariot que nous avons appliquée au platane étudié au $\S$ A.3.b à la hauteur $\mathrm{H}_{1}=0,73 \mathrm{~m}$.

La vitcsse du vent a été mesurée à l'aide d'un anémomètre électrodynamométrique MCB fixé sur un mât, à une hauteur égale à la hauteur moyenne du feuillage, le mât luimême étant placé à quelques mètres de l'arbre, perpendiculairement au lit du vent.

Pour assurer la simultanéité des prises de données, une caméra vidéo a enregistré sur une même image le cadran digital du voltmètre relié à l'anémomètre, le cadran du comparateur utilisé pour les déplacements du chariot et le cadran d'un chronomètre, à raison d'une image toutes les $0,02 \mathrm{~s}$ environ.

\section{Présentation des résultats}

Il est évident que l'intervalle de temps entre 2 acquisitions, imposé par les caractéristiques de la caméra, donne une précision illusoire, d'autant plus que le voltmètre numérique a scin propre temps de réponse de l'ordre de $0,35 \mathrm{~s}$. D'autre part, la réponse de l'arbre en début ou en fin de chaque turbulence n'est pas instantanée du fait de sa propre inertie. Il y a aussi un risque indiscernable d'entrée en résonance de l'arbre avec l'une des fréquences de la turbulence.

Des essais préliminaires où, par vent nul, l'arbre est soumis au moyen d'une corde à une force horizontale dont l'intensité est instantanément annulée, ont mis en évidence les oscillations du tronc dues à sa propre élasticité. Les 3 essais effectués montrent que le retour à l'équilibre nécessite $1,4 \mathrm{~s}$ environ en 2 oscillations amorties complètes, quelle que soit la valeur de la flèche avant interruption volontaire de l'effort.

Pour toutes ces raisons, les données brutes (fig. 8) ne donnent pas une relation bijective entre les 2 grandeurs mesurées. Il s'est avéré nécessaire de choisir sur les courbes des couples de points correspondant à un quasi-ćquilibre du fait de la faible variation de la vitesse du vent pendant un intervalle de temps de l'ordre de quelques dixièmes de seconde : maximum, monotonie d'un arc de courbe, etc...
Simultaneous recording of wind speed and tree bending at $H_{1}=0.73 \mathrm{~m}$.

Sur les 2 essais effectués, l'un par vent relativement faible, l'autre par vent légèrement plus turbulent, un certain nombre de couples de valeurs ont été retenues. Seuls sont présentés les résultats du $2^{\mathrm{e}}$ essai.

Nous avons déterminé la hauteur $\mathrm{H}$ du point d'application de la résultante de l'action du vent en faisant l'hypothèse que la charge du vent par unité de surface de l'obstacle est constante. Alors, le point d'application $G$ de

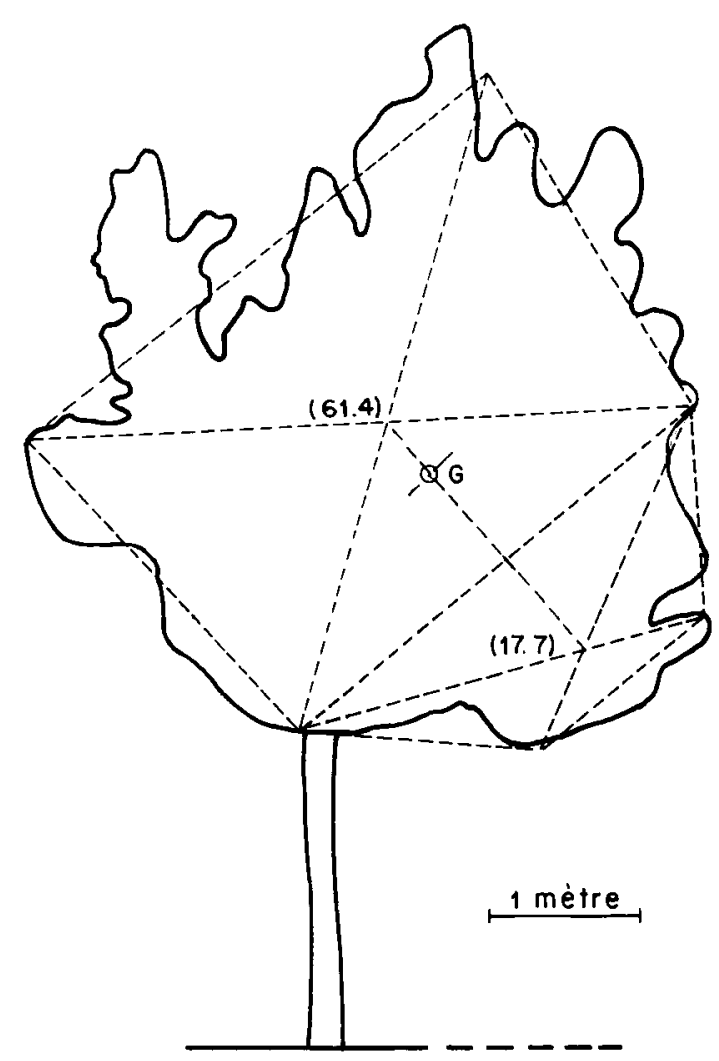

Figurc 9

Détermination simplifiée du centre de poussée.

Determination of wind pressure center. 
cette résultante est le centre de gravité de cette surface. Sur photo de la silhouette de l'arbre, ce centre de gravité a été déterminé par méthode graphique en réduisant le polygone la délimitant en 2 trapèzes (fig. 9). On considérera que la hauteur $\mathrm{H}$ est égale à $4 \mathrm{~m}$. Ainsi, avec :

$$
\begin{gathered}
\mathrm{H}=4 \mathrm{~m}, \mathrm{H}_{1}=0,73 \mathrm{~m}, \mathrm{~b}=0,16 \mathrm{~m}, \mathrm{R}=0,077 \mathrm{~m} \\
\text { donc } \quad \mathrm{I}=0,0276.10^{-3} \mathrm{~m}^{4} \text { et } \mathrm{E}=3,4 \mathrm{GPa}
\end{gathered}
$$

la relation théorique (1) s'écrit :

$$
y=24 \cdot 10^{-6} \mathrm{~F} \text {. }
$$

La comparaison des équations (5) et (6) montre que la flèche y est directement proportionnelle au carré de la vitesse du vent. Aussi les résultats des mesures expérimentales de ces 2 quantités ont-ils été portés (fig. 10) en coordonnées $\mathrm{V}$ et $\mathrm{y}^{1 / 2}$. Il vient :

$$
\mathrm{y}=121.10^{-6} \mathrm{~V}^{2} \text {. }
$$

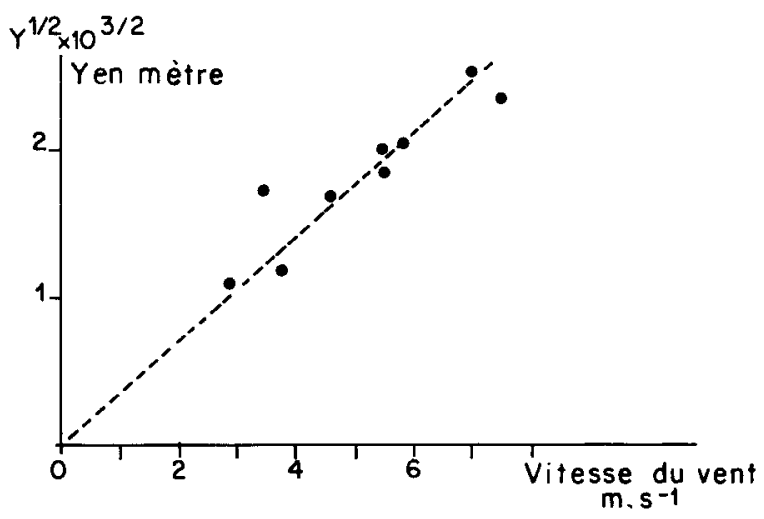

Figure 10

Relation entre la flèche d'un arbre et la vitesse du vent.

Trunk bending versus wind speed.

La détermination de la valeur numérique de l'aire $\mathrm{S}$ de la surface de la silhouette de l'arbre a été effectuée par découpage et pesée du support. Il a été obtenu $S=14 \mathrm{~m}^{2}$ ainsi, tous calculs faits, avec $\rho=1,3 \mathrm{~kg} \cdot \mathrm{m}^{-3}$, il vient :

$$
\mathrm{C}_{\mathrm{d}}=0,55
$$

soit $F=5 \mathrm{~V}^{2}$.

\section{Discussion}

La valeur du coefficient de trainée obtenue sur un jeune platane est compatible avec les résultats de RAYMER, présentés par GRACE (1977). Obtenues en soufflerie sur des essences de conifères, l'Auteur montre que les valeurs du coefficient de traînée (fig. 11) décroissent lorsque la vitesse du vent augmente. Aussi est-il nécessaire de préciser que la valeur de $0,55 \mathrm{de}$ nos essais est en fait une valeur moyenne dans la gamme de vitesses du vent mesurées, soit de 0 à $7 \mathrm{~m} . \mathrm{s}^{-1}$. On sait que la porosité de l'écran joue un rôle important dans la détermination de la valeur de $C_{d}$ (GUYOT, 1978), mais il est difficile d'imaginer la façon dont un pourrait déterminer la relation entre porosité de l'arbre et la vitesse du vent du fait de la mobilité du feuillage.

\section{Conclusion}

La notion de point d'ancrage équivalent que nous avons avancée a pu être mise concrètement en évidence à l'aide de
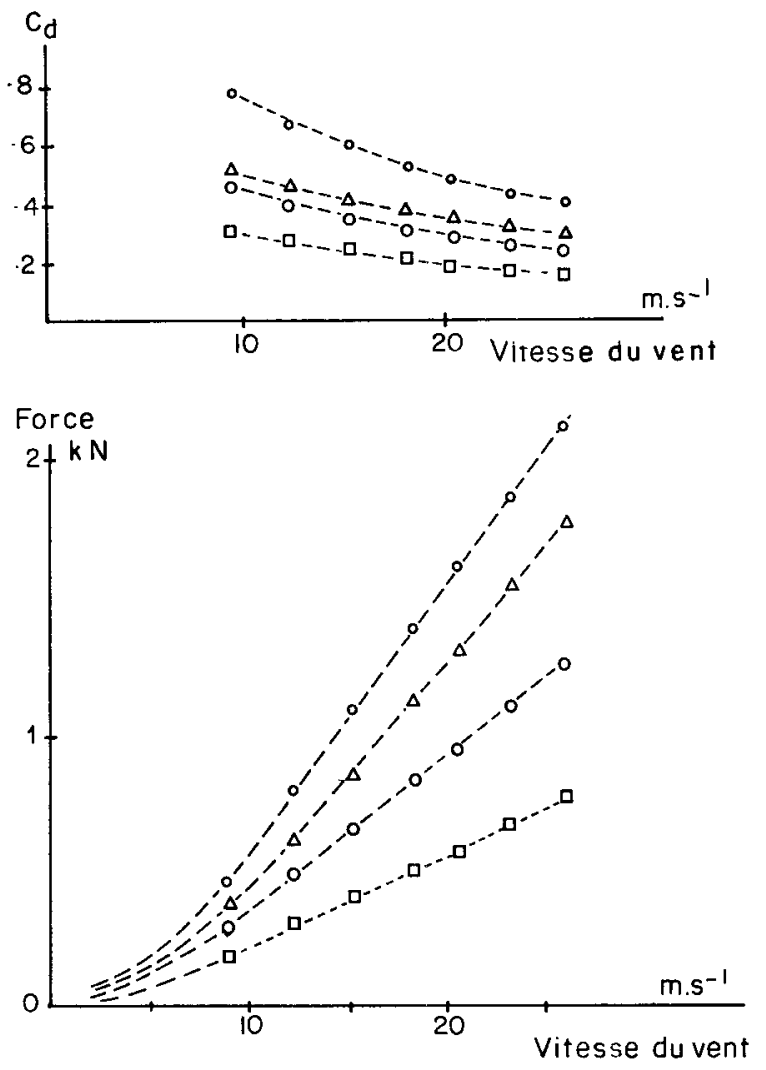

Figure 11

Coefficient de trainée et force agissant sur 4 espèces différentes de coniferes (selon RAYMER, in GRACE, 1977).

Drag coefficient and force acting on four species of conifer (after RAYMER in GRACE, 1977).

nos essais, bien que la démarche adoptée se soit heurtée à quelques difficultés expérimentales qui pourraient être levées dans l'avenir.

Les arbres qui ont été utilisés pour les mesures sont jeunes, de faible diamètre et de faible hauteur. La surface de l'obstacle qu'ils présentent au vent est petite. Leur point d'ancrage est peu profond. Toutefois, rares sont de tels arbres qui subissent des désordres irréversibles par l'action du vent.

Il semble évident que des arbres plus hauts, de surface d'obstacle plus importante ont un enracinement plus profond et plus important, augmentant par là même la profondeur du point d'ancrage. Ce sont pourtant ces arbres là qui sont le plus victimes du vent.

Ces premiers travaux ont montré qu'il est possible, par plusieurs méthodes utilisées simultanément, de définir les éléments de réduction des forces agissant sur un arbre lorsque le vent souffle :

- Intensité de l'effort horizontal,

- Hauteur du point d'application résultant de cet effort horizontal,

- Profondeur du point d'ancrage équivalent,

- Moment par rapport au point d'ancrage des efforts.

Il resterait maintenant à déterminer la façon dont cet ancrage est assuré par les racines et de déterminer les efforts maximaux auxquels elles peuvent résister tant par leurs propres caractéristiques mécaniques que par les caractéristiques du sol dans lequel elles se développent. 


\section{RÉFÉRENCES BIBLIOGRAPHIQUES}

Bean A., Alperi R. W., Federer C. A., 1975. A method for categorizing shelterbelt porosity. Agric. Meteorol., 14, 417-429.

Bucur V., 1981. Détermination du module d'Young du bois par une méthode dynamique sur carottes de sondage. Ann. Sci. For., 38 (3), 283-298.

Ferrand J. C., 1982. Un capteur d'extensométrie miniaturc à deux voies. Ann. Sci. For., 39 (2), 37-43.

Fraser A. I., Gardiner J. B. H., 1967. Rooting and stability in Sitka Spruce. Forestry Commission, Bull., $\mathrm{n}^{\circ} 40,27 \mathrm{p}$.

Grace J., 1977. Plant response to wind. Academic Press, 204 p.

Guyot G., 1978. Détermination des efforts exercés par le vent sur un brise-vent. Boundary-layer meteorology, 15, 57-67.
Hagen, L. J., Skidmore E. L., 1971a. Windbreak drag as influenced by porosity. Trans. ASAE, 14, 464-465.

Hagen L. J., Skidmore E. L., 1971b. Turbulent velocity fluctuations and vertical flow as affected by windbreak porosity. Trans. ASAE, 14, 634-637.

Plate E. J., 1971. The aerodynamics of shelter belts. Agric. Meteorol., 8, 203-222.

Seguin B., Gignoux N., 1974. Etude expérimentale de l'influence d'un réseau de brise-vent sur le profil vertical de vitesse du vent. Agric. Meteorol., 13, 15-23.

Somerville A., 1979. Root anchorage and root morphology of Pinus radiata on a range of ripping treatments. N.Z.J. For. Sci., 9 (3), 294-315. 